\title{
Scalings for a traveling mirror adiabatic magnetic compressor
}

\author{
P. M. Bellan \\ California Institute of Technology, Pasadena, California 91125 \\ (Received 7 December 1981; accepted for publication 29 March 1982)
}

Detailed practical scaling relations for a traveling mirror adiabatic magnetic compressor are derived, and an example is given of how this technique could be used to translate, compress, and heat the Los Alamos FRX-C reversed field theta pinch plasma.

PACS numbers: $52.55 . \mathrm{Ke}, 85.70 .-\mathrm{w}, 52.50 . \mathrm{Gj}$

\section{INTRODUCTION}

Controlled fusion will probably first be achieved in tokamaks. Tokamaks, however, have a number of features which make them unattractive for use as commercial fusion reactors, namely: tokamak geometry is complicated, the $\beta$ of a tokamak is low, and the minimum reactor size is large. Another magnetic confinement scheme, the reversed field configuration, has the closed field line geometry of a tokamak, but, unlike a tokamak, no mechanical structure linking the hole of the torus; hence, in principle, the very desirable confinement properties of a tokamak are obtained with a much simpler vacuum tank and external coil system. Substantial effort is being directed towards developing reversed field devices, namely: Astron-like electron or proton rings, ${ }^{1-4}$ reversed field theta pinches, ${ }^{5,6}$ and spheromaks. ${ }^{7-11}$

Because the reversed field plasma is not linked to a mechanical structure it is both translatable along its major axis, and almost arbitrarily compressible. The ability to be translated means that a reversed field fusion reactor could be split into distinct, linearly segmented sections, each optimized for a particular process (e.g., plasma or ring generation, fueling, heating, thermonuclear burning, exhaust); during the operating cycle the plasma would be moved from section to section. Segmented reactors of this sort have been proposed by Fleischmann and Kammash, ${ }^{12}$ and by Smith et al. ${ }^{13}$ The ability to be compressed without being blocked by a mechanical structure means that adiabatic compression and heating can be used to a much greater extent than possible for tokamaks. For example, the reactor design of Ref. 13 includes a compressor stage providing a $25 \times$ field compression ratio, which is far higher than what could be used in a tokamak reactor.

Recently the author proposed ${ }^{14-16}$ a novel scheme in which a traveling magnetic mirror "wave" simultaneously translates and compresses a field reversed configuration. Reference 14 described the principle of the scheme, Ref. 15 described how random gapped walls could be used to stabilize the traveling mirror against precessional (for particle rings) or radial shift (for plasmas) instabilities, and Ref. 16 showed an alternate approach for accomplishing the scheme of Ref. 14. The purpose of this paper is to provide detailed scaling relations from which practical devices can be constructed. The paper is organized as follows: Section I briefly reviews the concept, and Sec. II gives the derivation of the scaling relations, which are summarized in Table I.

\section{REVIEW OF THE TRAVELING MIRROR WAVE ADIABATIC COMPRESSOR ${ }^{14}$}

Figure 1 shows the essence of the scheme: by injecting a double-hump current pulse [Fig. 1(a)] into a macroscopic electronic delay line, a traveling magnetic mirror, i.e., a double-humped magnetic field, is made to propagate in wavelike fashion along the delay line. The actual delay line consists of solenoid coils connected to external capacitors as shown in Figs. 1(a) and (b). The direction of propagation is along the symmetry axis and the propagation velocity is determined by both the solenoid geometry and the external capacitance. This traveling magnetic mirror is used to carry a reversed field particle ring or compact torus in its trough (for a reversed field theta pinch a single humped propagating $B$ field could be used instead of a mirror field because the axial pinching selfforce of this plasma provides axial confinement).

By gradually changing the radius $r$, intercoil spacing $\Delta$, turns number $m$ and/or capacitance $C$, of successive coils in the solenoid delay line [as shown in Fig. 1(b)], the sectional delay time of successive delay line sections is made to be a slowly increasing function of axial position. Consequently, the propagation velocity becomes a slowly decreasing function of axial position. From the Wentzel-Kramers-Brillouin (WKB) theory, this causes the propagating magnetic mirror (or, for theta pinches, single hump) to steepen-i.e., its intensity increases and its axial extent decreases in a manner wherein the total magnetic energy remains constant (not counting particle diamagnetism). Thus, besides providing a simple way for achieving the moving field required for the reactors proposed in Refs. 12 and 13, this scheme provides adiabatic compression. The adiabatic compression is extremely efficient because, unlike conventional adiabatic compression, the volume occupied by the magnetic field shrinks along with the particle volume so no magnetic energy is wasted filling up unused volume. [Coensgen et al., ${ }^{17}$ 
noted this important point in the late 1950's and built a three-stage adiabatic compressor for low $\beta$ (i.e., not field reversed) mirror-confined plasmas, in which the third-stage magnetic field volume was $75 \times$ less than the first-stage volume. This device achieved $3 \mathrm{keV}$ ion temperatures; however, it was not MHD stabilized.]

The precise functional dependence of the WKB steepening on the coil parameters is derived in Refs. 14 and 16 , where it is shown that the magnetic field scales as

$$
B \sim \frac{m^{1 / 2} C^{1 / 4}}{\Delta^{3 / 4} r^{1 / 2}}
$$

and the characteristic mirror length $\lambda$ scales as

$$
\lambda \sim \frac{\Delta^{3 / 2}}{m r C^{1 / 2}}
$$

so that the magnetic energy $W_{B}$, which is proportional to $B^{2} r^{2} \lambda$, stays constant (not counting particle diamagnetism). The analysis of Refs. 14 and 16, and hence these scalings, is based on the assumptions that (i) $\lambda \gg r, \Delta$ and (ii) the coils are so closely spaced that each coil links more flux from adjacent coils than from itself (i.e. the coils act as a solenoid). Thus, to achieve an increasing field intensity having decreasing volume, all that is required is to gradually increase the quantity $m^{1 / 2} \mathrm{C}^{1 / 4} /$ $\Delta^{3 / 4} r^{1 / 2}$ for successive coils in the solenoid delay line while ensuring that the $\lambda \gg r, \Delta$ assumption always holds.

Various combinations of $m, C, r$, and $\Delta$ scalings are possible-the most obvious are (a) constant flux through the solenoid (axial contraction of the field volume without a change in radius would increase the flux): $B \sim r^{-2}, \lambda \sim r^{2}, \Delta \sim m^{2 / 3} C^{1 / 3} r^{2}$; (b) constant geometry (i.e., constant aspect ratio): $B \sim r^{-3 / 2}, \lambda \sim r, \Delta$ $\sim m^{2 / 3} C^{1 / 3} r^{4 / 3}$; (c) constant $T_{1} / T_{\|}$ratio for mirror confined particles (though not for field reversed configurations where the particles move in a field quite different from the external mirror field): $B \sim r^{-4 / 3}, \lambda \sim r^{2 / 3}, \Delta$ $\sim m^{2 / 3} C^{1 / 3} r^{10 / 9}$. For purposes of compressing reversed field configurations the constant geometry configuration is of most interest because it will not distort the aspect ratio of the reversed field plasma or particle ring. Section II gives the derivation of detailed scaling relations for designing practical constant geometry compressors.

\section{SCALING RELATIONS FOR PRACTICAL CONSTANT GEOMETRY COMPRESSORS}

In the following the compressor is assumed to consist of an initial large diameter straight segment connected to a tapered or "WKB" transition segment which in turn is connected to a final small diameter straight section (coil dimensions and capacitances are constant in the two straight segments). Particles are injected into (or are created in) the initial segment, are then translated into the WKB segment, where they are both translated and compressed, and then finally are translated into the small diameter segment. Appropriate scaling parameters are [here quantities are normalized to the initial (large end) radius, $r_{0}$, of the solenoid delay line]: $\alpha=r_{s} / r_{0}$, where (a)
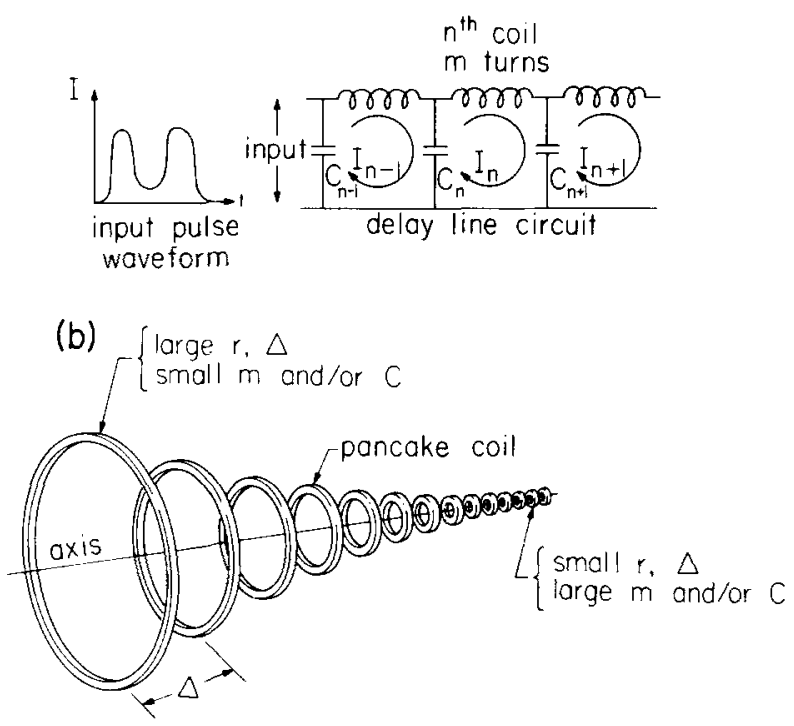

FIG. 1. (a) Input pulse and circuit; (b) coil geometry.

$r_{s}$ is the final radius; $C / r_{0}{ }^{3}$, where $C$ is the capacitance per section; $\delta / r_{0}$, where $\delta$ is the conductor radius; $\delta_{\text {sk }} / r_{0}$ where $\delta_{\text {sk }}$ is the skin depth; $t_{\text {comp }} / r_{0}^{2}$, where $t_{\text {comp }}$ is the characteristic time of the compressor; and $Z r_{0}$, where $Z$ is the characteristic delay line impedance.

Section IIA describes the relationships between the initial and final straight segments; these relationships give the main physical and electrical character of the compressor. Section IIB describes the WKB transition segment and gives scalings in terms of a WKB "gradualness" parameter $\epsilon$. Section IIC describes how compensation capacitors may be used to minimize phase velocity dispersion. Section IID describes how these relationships can be used to design a compressor, and Sec. IIE gives an example of a design.

\section{A. Scaling relationships between initlal and final compressor ends}

From Refs. 14 and 16 the fundamental relationships of a constant geometry compressor having a radial compression factor of $\alpha^{-1}$ are

$$
\begin{aligned}
& r \sim \alpha \\
& \Delta \sim m^{2 / 3} \alpha^{4 / 3} C^{1 / 3} \\
& B \sim \alpha^{-3 / 2} \\
& \lambda \sim \alpha \\
& I \sim m^{-1 / 3} \alpha^{-1 / 6} C^{1 / 3} .
\end{aligned}
$$

The associated electrical relations (in MKS units) are

$$
\begin{gathered}
B=\mu_{0} m I / \Delta, \\
L=\mu_{0} m^{2} \pi r^{2} / \Delta, \\
Z=\sqrt{(L / C),} \\
V=I Z,
\end{gathered}
$$




$$
\begin{gathered}
R=2 m \rho r / h \delta^{2}, \\
\delta_{\mathrm{sk}}=\left(2 t_{\text {comp }} / \mu_{0}\right)^{1 / 2},
\end{gathered}
$$

where $B$ is the magnetic field, $m$ is the turns per coil, $\Delta$ is the intercoil spacing, $r$ is the coil radius, $L$ is the effective coil inductance, $C$ is the capacitance per delay line section, $Z$ is the characteristic delay line impedance, $V$ is the voltage across the delay line, $R$ is the resistivity of a coil having $h$ parallel turns of a conductor with radius $\delta, \delta_{\text {sk }}$ is the skin depth, and $t_{\text {comp }}$ is the characteristic time of a pulse. Three constraints determine the scaling. (1) $W K B$ Requirement-The approximation $\lambda \gg \Delta, r$ must be satisfied at both the large (uncompressed) and the small (compressed) end, and the various parameters must satisfy Eqs. (1a)-(1e). (2) Space Limitations at Small End-For large compressions the small end requires densely packed coils; the space available at the small end places an upper limit on the turns available for a given size conductor. (3) Avoidance of Dissipative Attenuation-The coils have a finite resistance which causes damping of the propagating field; at high frequencies the effective resistance is higher because the current is confined to a narrow skin depth. The coils must be designed so that this damping is negligible. These constraints will now be used to obtain the compressor scaling relations.

\section{WKB requirements}

The optimum choice for the intercoil spacing $\Delta$ is $\Delta$ $=r$, since $\Delta \gg r$ would lead to bumpy fields, while $\Delta$ $\ll r$ would increase the device complexity but would not improve the field smoothness relative to $\Delta=r$. The WKB requirement reduces to $\lambda \gg \Delta=r$ for both the small and large ends. Equation ( $1 \mathrm{~b})$ then gives

$$
\frac{\Delta_{s}}{\Delta_{0}}=\left(\frac{m_{s}}{m_{0}}\right)^{2 / 3}\left(\frac{r_{s}}{r_{0}}\right)^{4 / 3}\left(\frac{C_{s}}{C_{0}}\right)^{1 / 3},
$$

where a subscript " 0 " denotes large end quantities and a subscript " $s$ " denotes small end quantities. Using $\Delta_{s}$ $=\alpha r_{0}$ this becomes

$$
m_{s} C_{s}^{1 / 2}=m_{0} C_{0}^{1 / 2} \alpha^{-1 / 2} .
$$

By choosing $\lambda_{s}=10 r_{s}$ the $\lambda \gg r, \Delta_{s}$ requirement is satisfied at the small end and from Eqs. (1a) and (1d) this gives

$$
\lambda_{0}=10 r_{0} .
$$

(The factor 10 is chosen because the hills and valley of a traveling mirror are typically one third the overall length $\lambda$ of the mirror.) The characteristic time $t_{\text {comp }}$ of a pulse is the product of the number of delay line sections and the time per section $\sqrt{L_{0}} C_{0}$. Thus

$$
t_{\mathrm{comp}}=\frac{\lambda_{0}}{\Delta_{0}} \sqrt{L_{0} C_{0}}=10 m_{0} r_{0}^{2} \sqrt{\mu_{0} \pi C_{0} / r_{0}^{3}}
$$

or

$$
t_{\text {comp }}=2 \times 10^{-2} m_{0} r_{0}^{2} \sqrt{C / r_{0}{ }^{3}} .
$$

$t_{\text {comp }}$ is invariant during compression-the same $t_{\text {comp }}$ would be calculated using small end parameters. Often one wishes to specify $t_{\text {comp }} / r_{0}^{2}$ and then calculate $m_{0}^{2} C_{0} /$ $r_{0}^{3}$, in which case Eq. (5) can be written as

$$
m_{0}^{2} C_{0} / r_{0}^{3}=2.5 \times 10^{3}\left(t_{\text {comp }} / r_{0}^{2}\right)^{2}
$$

\section{Space limitations at small end}

The reduced volume at the small end of the compressor places an upper bound on the number of turns possible on the small end coils. In calculating this number it is assumed that $\delta_{\text {sk }}>\delta$-otherwise all the current would flow in a small surface layer of the conductor so that one would employ the available volume more efficiently by using a larger number of smaller diameter conductors.

The maximum possible cross section of a coil at the small end is determined by the requirement that the coil width be slightly less than the intercoil spacing, and the coil radial thickness be much less than the coil radius (otherwise the effective coil radius would change). Assuming the coil width to be $\Delta_{s} / 2$ and the coil thickness to be $r_{s} / 5$, the maximum allowed number of turns on the small end of the coil is then

$$
m_{s} \leq \frac{\text { coil cross section }}{\text { conductor cross section }}=\frac{\Delta_{s}{ }^{2}}{10 \pi \delta^{2}}=\alpha^{2} \frac{r_{0}{ }^{2}}{10 \pi \delta^{2}} .
$$

The above expression can be combined with Eq. (3) to give an upper bound on the conductor radius

$$
\left(\frac{\delta}{r_{0}}\right)^{2} \leq \frac{\alpha^{5 / 2}}{10 \pi m_{0}}\left(\frac{C_{s}}{C_{0}}\right)^{1 / 2}
$$

or

$$
\left(\frac{\delta}{r_{0}}\right)^{2} \leq 0.03 \frac{\alpha^{5 / 2}}{m_{0}}\left(\frac{C_{s}}{C_{0}}\right)^{1 / 2} \text {. }
$$

The skin depth assumption discussed above provides an additional upper bound on the conductor radius; using Eq. (2f) and $\delta_{\text {sk }}>\delta$, this is

$$
\left(\frac{\delta}{r_{0}}\right)^{2}<\frac{2 \rho t_{\text {comp }}}{\mu_{0} r_{0}^{2}}
$$

or for $20^{\circ} \mathrm{C}$ copper $\left(\rho=1.7 \times 10^{-8} \Omega \mathrm{m}\right)$

$$
\left(\frac{\delta}{r_{0}}\right)^{2}<0.03 \frac{t_{\text {comp }}}{r_{0}{ }^{2}} \text {. }
$$

\section{Avoidance of dissipative attenuation}

The following simple dispersion can be associated with the solenoid delay line wave equation:

$$
\frac{\omega^{2}}{k^{2}}=\frac{\Delta^{2}}{L C} \text {. }
$$

If finite coil resistance is included then $i \omega L \rightarrow i \omega L+R$. The damping can be described by an imaginary part of $k$ so that the dispersion relation becomes

$$
\frac{\left(k_{r}+i k_{i}\right)^{2}}{\omega^{2}}=\frac{(L-i R / \omega) C}{\Delta^{2}} .
$$

If $\omega L \gg R$, the damping is small-i.e., $k_{i} \ll k_{r}$ - and $k_{i}$ 
is given by

$$
k_{i} \Delta=\frac{R}{2 \sqrt{L / C}}=\frac{R}{2 Z}=\frac{1}{2 Q},
$$

where $\left.Q=\sqrt{\left(L / R^{2}\right.} C\right)$ is the quality factor per section. Negligible damping of the propagating field will be obtained if $N k_{i} \Delta<0.1$, where $N$ is the number of coil sections. Since $Q$ can be different for the small and large ends it is necessary to consider the small and large ends separately.

At the small end $N=10$, so that $Q_{s}>50$ is required. From Eqs. (2b) and (2e) the requirement on $Q_{s}$ may be expressed as

$$
Q_{s}=\frac{\sqrt{\mu_{0} \pi}}{2 \rho} \alpha^{-1 / 2}\left(\frac{\delta}{r_{0}}\right)^{2}\left(\frac{C_{s}}{r_{0}}\right)^{-1 / 2}>50,
$$

or using Eqs. (3) and (6), and assuming $20^{\circ} \mathrm{C}$ copper conductors,

$$
0.04 \frac{t_{\text {comp }}}{m_{s} r_{0}^{2}}<\left(\frac{\delta}{r_{0}}\right)^{2}
$$

At the large end, for convenience of calculation, we assume that the same size conductor is used as at the small end, but that there now are $h$ parallel conductors. In practice a single larger radius conductor would be used since there is plenty of space at the large end. Since the large end also has 10 sections the requirement for avoidance of damping is again

$$
10 k_{i} \Delta>50 \text { or } Q_{0}>50 .
$$

Because of the difference in coil dimensions between the large and small ends the $Q$ 's are different and in particular

$$
Q_{0}=h \alpha^{1 / 2}\left(C_{s} / C_{0}\right)^{1 / 2} Q_{s} .
$$

Since $Q_{s}$ was arranged to be $>50$, the large end requirement becomes simply

$$
h>\alpha^{-1 / 2}\left(C_{s} / C_{0}\right)^{-1 / 2} .
$$

The characteristic delay line impedance, the magnetic field, and the stored energy will now be calculated. From Eq. (2c) the large end impedance is

$$
\begin{aligned}
Z_{0}=\left(\mu_{0} \pi\right)^{1 / 2} m_{0} r_{0}{ }^{-1} & \left(C_{0} / r_{0}{ }^{3}\right)^{-1 / 2} \\
& =2 \times 10^{-3} m_{0} r_{0}^{-1}\left(C_{0} / r_{0}{ }^{3}\right)^{-1 / 2},
\end{aligned}
$$

while from Eqs. (2c) and (3) the small end impedance is

$$
Z_{s}=\left(m_{s} / m_{0}\right)^{2} \alpha Z_{0} .
$$

It is most convenient to express the magnetic field strength in terms of voltage since voltage breakdown limitations are typically more stringent than current limitations. From Eqs. (2a), (2d), and (11) the large end magnetic field is

$$
B_{0}=\sqrt{\frac{\mu_{0}}{\pi}}\left(\frac{C_{0}}{r_{0}^{3}}\right)^{1 / 2} V_{0}
$$

or in terms of $t_{\text {comp }} / r_{0}^{2}$ [using Eq. (6)]

$$
B_{0}=3 \times 10^{-2} \frac{t_{\text {comp }}}{m_{0} r_{0}{ }^{2}} V_{0} .
$$

(Note that this is an expression giving flux in terms of applied volt-seconds). From Eq. (1c) the small end field is a factor $\alpha^{-3 / 2}$ higher.

The stored magnetic energy is

$$
W_{B}=\frac{B_{0}^{2}}{2 \mu_{0}} \pi r_{0}^{2} \lambda_{0}=5 C_{0} V_{0}^{2}=\frac{B_{s}^{2}}{2 \mu_{0}} \pi r_{s}^{2} \lambda_{s}=5 C_{s} V_{s}^{2},
$$

while the total stored energy (magnetic energy plus energy stored in the capacitors) is

$$
W_{\text {total }} \doteq \frac{V_{0}^{2}}{Z_{0}} t_{\text {comp }}
$$

or simply

$$
W_{\text {total }}=10 C_{0} V_{0}^{2}
$$

using Eqs. (2c), (2b), and (5). Thus the stored capacitive energy equals the stored magnetic energy, which is to be expected for a propagating wave.

\section{B. The "WKB'" transition segment}

The transition is designed so that (i) the WKB criterion of $k^{-1} d k / d z \ll k$ is locally satisfied at each delay line section (here $k$ is the wavenumber of the longest axial Fourier component making up the traveling mirror, i.e., $k=\pi / \lambda$, where $\lambda$, as before, is the axial length of the traveling mirror), and (ii) each delay line section in the transition also locally satisfies Eqs. (1a)-(1e).

The WKB criterion can be written quantitatively as

$$
k^{-2} d k / d z=\epsilon,
$$

where $\epsilon$ is a measure of the "gradualness" of the taper and $\epsilon<1$; for constant $\epsilon$ (which is assumed here) Eq. (12) may be integrated to give

$$
\frac{1}{k(z)}=\frac{1}{k(0)}-\epsilon z
$$

here $z=0$ refers to the beginning of the transition. In terms of $\lambda$ this may be expressed as

$$
\lambda(z)=\lambda_{0}\left(1-\epsilon \pi z / \lambda_{0}\right) .
$$

Defining $\alpha_{\text {loc }}(z)=r(z) / r_{0}=\lambda(z) / \lambda_{0}$ to be the local value of $\alpha$ in the transition section [i.e., $\alpha_{\mathrm{loc}}(0)=1$ and $\alpha_{\mathrm{loc}}\left(z_{\max }\right)=\alpha$, where $z_{\max }$ is the length of the transition section], Eq. (12) can be written as

$$
\alpha_{\mathrm{loc}}(z)=\left(1-\epsilon \pi z / \lambda_{0}\right)
$$

and also

$$
z_{\max }=(1-\alpha) \frac{\lambda_{0}}{\epsilon \pi} .
$$

Another quantity of interest is the number of delay line sections $N$ in the transition; this is simply

$$
N=\int_{0}^{z_{\max }} \frac{d z}{\Delta(z)} .
$$




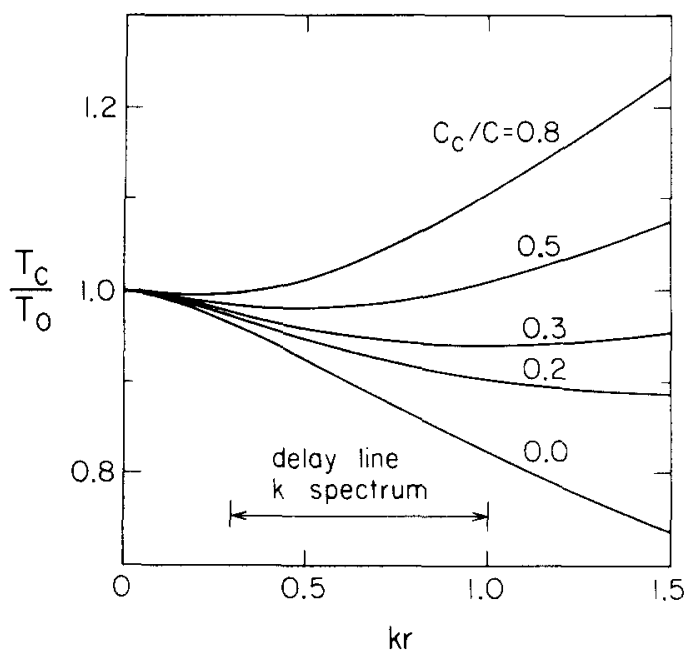

Fig. 2. Time delay (normalized to $k=0$ delay) vs wavenumber for various values of compensation capacitance (normalized to delay line capacitance)

Assuming $\Delta(z)=r(z)$ as in the initial and final ends of the compressor, this becomes

$$
N=\frac{10}{\epsilon \pi} \ln \left(\alpha^{-1}\right)
$$

Finally, $m$ and $C$ for delay line sections in the transition is given by extension of Eq. (3) as

$$
m(z)^{2} C(z)=m_{0}{ }^{2} C_{0} \alpha_{100}{ }^{-1} .
$$

\section{Compensation to minimize phase velocity dispersion}

Until now it has been assumed that the wave propagation on the delay line is free from phase velocity dispersion. In fact, there is such a dispersion because, due to the fact that large $k$ modes involve fewer adjacent coils than do small $k$ modes, the effective coil inductance is a function of $k$. Equation ( $2 \mathrm{~b})$, which is the inductance of an infinitely long solenoid, is only exact for $k=0$. Blewett and Rubel ${ }^{18}$ reported an unpublished expression derived by Poritsky and Blewett for the finite $k$ inductance of a straight (i.e., nontapered) delay line. This finite $k$ inductance is ${ }^{17}$

$$
L=L_{0}\left[2 K_{1}(k r) I_{1}(k r)\right],
$$

where $L_{0}$ is the $k=0$ inductance from Eq. (2b) and $K_{1}$, $I_{1}$ are modified Bessel functions. Because large $k$ modes involve fewer coils, the inductance given by Eq. (17) is a monotonically decreasing function of $k$; consequently, larger $k$ 's will have a shorter propagation time $\left[\sim(L C)^{1 / 2}\right]$. Erickson and Sommer ${ }^{19}$ showed that by inserting small "compensation" capacitors in parallel with the coils this decrease of inductance with frequency can, to some extent, be cancelled because the effective inductance $L_{\text {eff }}$ then becomes

$$
L_{\mathrm{eff}}=\frac{L}{1-\omega^{2} L C_{c}},
$$

where $\omega$ is the mode frequency and $C_{c}$ is the compensation capacitance. Defining the compensated delay time per section as $T_{c}=\left(L_{\mathrm{eff}} C\right)^{1 / 2}$, and the $k=0$ delay time as $T_{0}=\left(L_{0} C\right)^{1 / 2}$, and noting that $\omega / k=\Delta / T_{c}$, the compensated delay can be expressed as ${ }^{19}$

$$
\frac{T_{c}}{T_{0}}=\left\{\left[1+k^{2} \Delta^{2} C_{c} / C\right] 2 K_{1}(k r) I_{1}(k r)\right\}^{1 / 2} .
$$

Thus, the monotonic increase with respect to $k$ of the compensation factor can be used to offset the monotonic decrease of the $I_{1}(k r) K_{1}(k r)$ factor. Because the two factors are not exact inverses, this balancing will be $k$ dependent. Erickson and Sommer gave a plot of Eq. (19) for various $C_{c} / C$; Fig. 2 reproduces their plot for the case $r=\Delta$ which is being assumed here. From this plot and a consideration of the $k$ spectrum of the traveling mirror it is possible to choose the optimum amount of compensation. In particular, the traveling mirror compressor design described in the previous sections had $\Delta=r$, an overall mirror length of $10 r$ (this gives the half-wavelength of the longest Fourier mode), and mirror peaks having axial length of about $2-3 r$ (this gives the halfwavelength of the shortest Fourier mode in the spectrum). Hence, the traveling mirror spectrum is $0.3<k r$ $<1$. This spectral range is indicated at the bottom of Fig. 2, and a comparison with the curves shows that a good choice of compensation for this range is $C_{c} / C$ $\sim 0.5$. Compensation has been found to work very well on model compressors constructed by the author. Without compensation, the waveform develops "wiggles" due to interference between the dispersive Fourier modes, whereas with compensation the waveform is undistorted.

\section{Designing a compressor}

Table I summarizes the constant geometry scaling relations derived in Sec. IIA-C. To design a compressor one typically chooses $r_{0}$ to give the overall physical dimensions; $t_{\text {comp }}$ to match the lifetime of what is being compressed; and $\alpha$, the desired compression ratio. From these input parameters Table I provides the required capacitance- turns-number combination, the magnetic field strength per applied volt, the impedance, and the amount of field compression. As noted in Ref. 16, either the turns number or the capacitance can be made the gradually changing parameter. If the turns number is made the gradually changing parameter, then rather large turns numbers are required (because turns number is quantized) giving high impedances, and high voltages. Changing $C$ instead, allows low turns number to be used so that a low-impedance compressor can be built.

The allowed parameter regimes are bounded by the need to avoid resistive damping, by skin depth restraints, and by space limitations for the conductors at the small end of the compressor. These requirements are quantitatively summarized by the bounds on the normalized conductor radius given in Table I(b). The lower bound corresponds to avoiding resistive damping, the first upper bound to space limitations at the small end (dominant when $t_{\text {comp }}$ is long) and the second upper bound to skin 
(a) Independent variables Initial radius

Initial turns number or initial capacitance

Characteristic compression time

Radial compression factor

$$
\begin{aligned}
& r_{0} \\
& m_{0} \\
& \text { or } C_{\mathbf{0}} \\
& t_{\text {comp }} \\
& \alpha^{-1}
\end{aligned}
$$

(b) Dependent parameter ratios

\section{Capacitance, turns number}

$m_{0}^{2} \frac{C_{0}}{r_{0}^{3}}=2.5 \times 10^{3}\left(\frac{t_{\text {comp }}}{r_{0}^{2}}\right)^{2}$

Conductor (copper) radius

$$
\frac{0.04 t_{\text {comp }}}{m_{s} r_{0}^{2}}<\left(\frac{\delta}{r_{0}}\right)^{2}<\min \left[\frac{0.03 \alpha^{5 / 2}}{m_{0}}\left(\frac{C_{s}}{C_{0}}\right)^{1 / 2}, \frac{0.03 t_{\text {comp }}}{r_{0}^{2}}\right]
$$

(c) Stored energy

$$
W_{B}=\frac{B_{0}^{2}}{2 \mu_{0}} \pi r_{0}^{2} \lambda_{0}=5 C_{0} V_{0}^{2}
$$

$W_{\text {total }}=2 W_{B}$

(d) Uncompressed vs Compressed Parameters

\begin{tabular}{llll}
\multicolumn{1}{c}{ Quantity } & \multicolumn{1}{c}{ Ratio } & Initital Value & Final Value \\
\hline Coil bore, intercoil spacing & $r / r_{0}, \Delta / \Delta_{0}$ & 1 & $\alpha$ \\
Mirror length & $\lambda / r_{0}$ & 10 & $10 \alpha$ \\
Turns per coil, capacitance & $m C^{1 / 2} / m_{0} C_{0}^{1 / 2}$ & 1 & $\alpha^{-1 / 2}$ \\
No. of parallel conductors & $\mathrm{h}$ & $\alpha^{-1 / 2}\left(C_{d} / C_{s}\right)^{1 / 2}$ & 1 \\
Delay line impedance & $Z r_{0}$ & $0.002 m_{0}\left(C_{0} / r_{0}^{3}\right)^{1 / 2}$ & $\alpha\left(m_{s} / m_{0}\right)^{2} Z_{0} r_{0}$ \\
Magnetic field & $B$ & $0.03 t_{\text {comp }} V_{0} / m_{0} r_{0}^{2}$ & $\alpha^{-3 / 2} B_{0}$
\end{tabular}

(e) WKB transition segment ( $z=0$ refers to beginning of this segment)

$\begin{array}{ll}\text { Gradualness factor } & \epsilon=k^{-2} d k / d z \\ \text { Local } \alpha & \alpha_{100}(z)=\left(1-\epsilon \pi z / \lambda_{0}\right) \\ \text { Local } m^{2} C & m^{2} C=m_{0}^{2} C_{0} \alpha_{10 c}^{-1} \\ \text { Overall length } & z_{\max }=(1-\alpha) \frac{\lambda_{0}}{\epsilon \pi} \\ \text { Number of sections } & N=\frac{10}{\epsilon \pi} \ln \left(\alpha^{-1}\right)\end{array}$

depth limitations (dominant when $t_{\text {comp }}$ is short). Table I also shows that operation at short characteristic times requires high voltages (since $B r^{2} \sim V_{0} t_{\text {comp }}$ ). Table I can also be used for building scale models of large devices so that designs can be tested inexpensively. For this purpose one designs the model to have the same $C / \tau_{0}{ }^{3}, t_{\text {comp }} /$ $r_{0}^{2}, Z r_{0}, \delta / \tau_{0}, \delta_{\text {sk }} / r_{0}$, and turns numbers as the large device. By making these ratios the same, the small device operates in the same nondimensional parameter regime (i.e., same skin depth, resistive damping, and space limitations) as the large device, and also requires the same applied voltage for a given $B$. As the scalings show, $C$, $t_{\text {comp }}$, and $\delta$ are all smaller for the model, while its impedance is larger.

In summary, Table I shows how to build practical devices of arbitrary size. In general, larger devices have longer characteristic times, require bigger capacitors, and have more stored energy than smaller devices, while for a given sized device, operation at short characteristic times requires high voltages.

\section{E. An application: Compression and heating of the Los Alamos FRX-C plasma}

A large reversed field theta pinch, FRX-C, is now beginning operation at Los Alamos. The design parameters $\operatorname{are}^{20}$

plasma $\begin{cases}\text { length } & 70 \mathrm{~cm} \\ \text { radius } & 9 \mathrm{~cm} \\ \text { density } & 3 \times 10^{15} \mathrm{~cm}^{-3} \\ T_{e}, T_{i} & 500 \mathrm{eV} \\ \text { total particle energy } & 8.4 \mathrm{~kJ} \\ \text { lifetime } & 100-200 \mu \mathrm{s}\end{cases}$
confining field $\begin{cases}\text { magnitude } & 11 \mathrm{kG} \\ \text { length } & 225 \mathrm{~cm} \\ \text { radius } & 22.5 \mathrm{~cm}\end{cases}$

Starting with these parameters it is straightforward to design a traveling wave compressor which would heat and compress this plasma substantially. The compressor parameters would be the following:

compressor $\begin{cases}\tau_{\text {comp }} & 50 \mu \mathrm{S} \\ \alpha & 0.4 \\ \text { magnetic energy } & 190 \mathrm{~kJ}\end{cases}$
initial plasma
$\begin{array}{ll}\text { length } & 70 \mathrm{~cm} \\ \text { radius } & 9 \mathrm{~cm} \\ \text { density } & 3 \times 10^{15} \mathrm{~cm}^{-3} \\ \text { total particle energy } & 8.4 \mathrm{~kJ}\end{array}$


large end small end

confining field diameter

confining field length

$45 \mathrm{~cm}$

$225 \mathrm{~cm}$

$18 \mathrm{~cm}$

coil turns

2

impedance

$0.72 \Omega$

$6.9 \mu \mathrm{F}$

$75 \mathrm{kV}$

$11 \mathrm{kG}$

$0.5 \mathrm{keV}$

$0.5 \mathrm{keV}$

$8.4 \mathrm{~kJ}$
$90 \mathrm{~cm}$

2

$0.29 \Omega$

$17 \mu \mathrm{F}$

$47 \mathrm{kV}$

$43 \mathrm{kG}$

$2.0 \mathrm{keV}$

$2.0 \mathrm{keV}$

$34 \mathrm{~kJ}$ total particle energy

The coil geometry of such a compressor is shown in Fig. 3(a), and the propagation and compression of a magnetic field for one particular choice of input waveform is shown in Figs. 3(b)-(d). The WKB parameter for the transition section has been chosen to be $\epsilon=0.2$, so that from Eqs. (14) and (15) the number of coils in the transition section is 14 and the length of the transition section is $2.1 \mathrm{~m}$. To keep the voltages from being too high, the compressor is a variable capacitance design having only two turns per coil; this low turns number has the further advantage of allowing high mechanical strength windings necessary to withstand the large magnetic forces (the pressure of the compressed magnetic field is $\sim 70$ atm).

It should be pointed out that the usual physics obstacles (e.g., stability, radiation, wall bombardment) would have to be overcome to make this design work; however, these are not peculiar to this scheme, and will have to be solved for virtually any approach. The essential feature of this design is that it provides a simple, efficient,

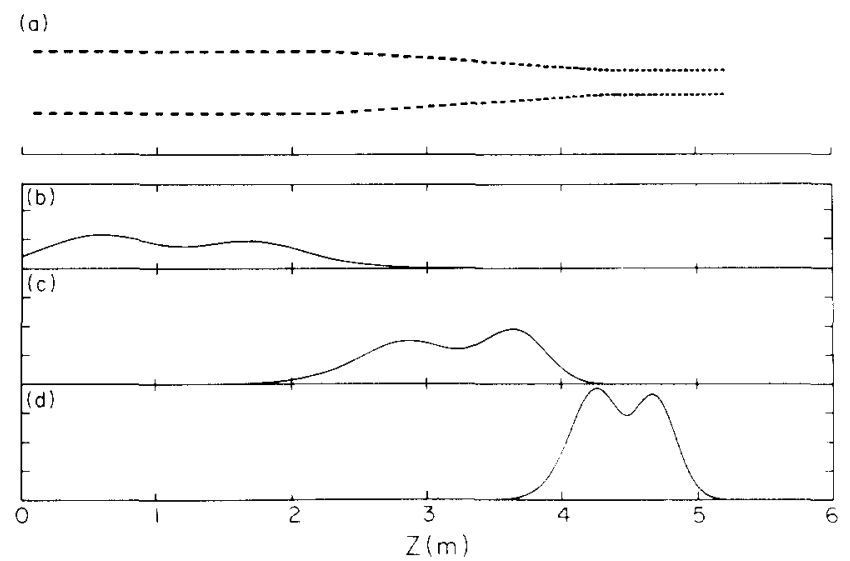

FIG. 3. (a) Coil geometry of compressor scaled for FRX-C reversed field theta pinch. (b)-(d) Propagation and compression of typical traveling field at 0,50 , and $100 \mu \mathrm{s}$, respectively.

and credible way of substantially heating and compressing the FRX-C plasma.

The analysis in this paper does not consider the effect of the plasma back on the compressor, and so is valid only for situations where the compressed plasma energy is much less than the total magnetic energy (this is a limitation of the analysis, not necessarily of the conceptconsideration of high $\beta$ effects would require a much more sophisticated theory). The example presented in this section has accordingly been chosen, so that the compressed plasma energy is $\sim 20 \%$ of the total magnetic energy.

\section{ACKNOWLEDGMENT}

The author is an Alfred P. Sloan Foundation Fellow.

N. C. Christofilos, Proceedings of the 2nd U.N. Conf. on Peaceful Uses of Atomic Energy (United Nations, Geneva, 1958), Vol. 32, p. 279 .

${ }^{2}$ M. L. Andrews, H. Davitian, H. H. Fleischmann, B. Kusse, R. E. Kribel, and J. A. Nation, Phys. Rev. Lett. 27, 1428 (1971).

${ }^{3}$ C. A. Kapetanakos, J. Golden, J. A. Pasour, S. J. Marsh, and R. A. Mahaffey, Phys. Rev. Lett. 44, 1218 (1980).

${ }^{4}$ J. B. Greenly, P. L. Dreike, D. A. Hammer, P. M. Lyster, Y. Nakagawa, and R. N. Sudan, Proceedings of the Third Symposium on Compact Toroids (Los Alamos Scientific Laboratory, Los Alamos, NM, 1980), Report No. LA-8700-C.

${ }^{5}$ A. G. Esk'ov, R. Kh. Kurtmullaev, A. P. Kreshchuk, Ya. N. Laukhin, A. I. Malyutin, A. I. Markin, Yu. S. Martyushov, B. N. Mirinov, M. M. Orlov, A. P. Proshletsov, V. N. Semenov, and Ya. B. Sosunov, in Plasma Physics and Controlled Nuclear Fusion Research (International Atomic Energy Agency, Vienna, 1978), Vol. II, p. 187

${ }^{6}$ W. T. Armstrong, R. K. Linford, J. Lipson, D. A. Platts, and E. G. Sherwood, Phy. Fluids, 24, 2068 (1981).

${ }^{3}$ H. Alfven, L. Lindberg, and P. Mitliel, J. Nucl. Energy Part C 1, $116(1960)$.

${ }^{8}$ T. R. Jarboe, I. Henins, H. W. Hoida, R. K. Linford, J. Marshall, D. A. Platts, and A. R. Sherwood, Phys. Rev. Lett. 45, 1264 (1980).

${ }^{9}$ W. C. Turner, G. C. Goldenbaum, E. H. A. Granneman, C. W. Hartman, D. S. Prono, J. Taska, and A. C. Smith, Proceedings of the Third Symposium on Compact Toroids (Los Alamos Scientific Laboratory, Los Alamos, NM, 1980), Report No. LA-8700-C.

${ }^{10}$ M. Yamada, H. P. Furth, W. Hsu, S. Jardin, A. Janos, M. Okabayashi, J. Sinnis, T. H. Stix, K. Yamazaki, Phys. Rev. Lett. 46, $188(1981)$.

" G. C. Goldenbaum, J. H. Irby, Y. P. Chong, and G. W. Hart, Phys. Rev. Lett. 44, 393 (1980).

${ }^{12}$ H. H. Fleischmann and T. Kammash, Nuc. Fusion 15, 1143 (1975).

${ }^{13}$ A. C. Smith, G. A. Carlson, H. H. Fleischmann, W. Grossman Jr., T. Kammash, K. R. Schultz, and D. M. Woodall, Proceedings Third Symposium on Compact Toroids (Los Alamos Scientific Laboratory, Los Alamos, NM, 1980), Report No. LA-8700-C.

${ }_{14}$ P. M. Bellan, Phys. Rev. Lett. 43, 858 (1979).

${ }^{15}$ P. M. Bellan, Phys. Fluids, 24, 947 (1981).

${ }^{16}$ P. M. Bellan, Phys. Fluids 24, 2128 (1981).

${ }^{17}$ F. H. Coensgen, W. F. Cummins, A. E. Sherman, Phys. Fluids 2, 350 (1959)

18 J. P. Blewett and H. H. Rubel, Proc. IRE 35, 1580 (1947).

${ }^{19}$ R. A. Erickson and H. Sommer, Proc. IRE 38, 1036 (1950).

${ }^{20}$ R. E. Siemon and R. R. Bartsch, Proceedings of the Third Symposium on Compact Toroids (Los Alamos Scientific Laboratory, Los Alamos, NM, 1980), Report No. LA-8700-C. 